\title{
What Matters Most? Students' Rankings of Simulation Components That Contribute to Clinical Judgment
}

\author{
Michelle A. Kelly, MN, RN; Paul Hager, PhD; and Robyn Gallagher, PhD, RN \\ Ms. Kelly - Senior Lecturer and Director, Simulation and Technologies, Faculty of Health, \\ Dr. Hager - Emeritus Professor, Learning Cultures and Practices Group, Faculty of Arts and Social Sciences \\ Dr. Gallagher - Associate Professor, Chronic and Complex Care, and Director, Research Students, Faculty of Health \\ University of Technology, Sydney, New South Wales, Australia.
}

\begin{abstract}
As the pedagogy of healthcare simulation matures there is increasing variation in the level of guidance provided and types of simulation components included. To prepare students for professional practice, one university embedded Tanner's model of clinical judgement within the nursing curricula and integrated simulations. There was interest in seeking students' opinions of 'what matters most' in the design and delivery of simulations which may vary from the academic's viewpoint. Senior undergraduate nursing students $(\mathrm{N}=150)$ from three types of study programs rated 11 simulation components in relation to clinical judgement. The three student groups rated all components above 2.9 on a five-point Likert scale with some variation across groups for component rankings. Highest ranking components for applying clinical judgement were facilitated debriefing; post simulation reflection and guidance by the academic. Lowest ranked components were patient case notes and orientation to the simulation area. Age and previous nursing experience did not influence study variables.
\end{abstract}

\section{Introduction}

Contemporary simulation activities within highly authentic environments are popular learning strategies across health care professions, at undergraduate level and for continuing professional development. For undergraduate students, a common aim of simulation is to provide a holistic learning opportunity, which merges theory with clinical practice perspectives, often providing insight into the professional role and expected behaviours (McGrath, Lyng, \& Hourican, 2012; 
Ricketts, 2011). The importance of teamwork and effective communication are other aims frequently included as simulation learning outcomes (Laschinger et al., 2008; Miller, Riley, Davis, \& Hansen, 2008). There is much literature to support these foci as important objectives for simulation learning encounters (McGaghie, Issenberg, Petrusa, \& Scalese, 2010; Neill \& Wotton, 2011; Schlairet, 2011). However, despite best intentions, there may be a mismatch between the planned benefits, participants' perceptions of learning, and learning outcomes following the simulation encounter.

Much time and effort are expended when creating simulation encounters to achieve the aforementioned aims. In a systematic review of twelve selected studies, Cant and Cooper (2010) summarised core components included in effective simulations as briefing and orientation to the environment and simulator, the simulation activity and a debriefing session. These are considered the minimal elements to provide a safe, meaningful simulation learning encounter (Arthur, LevettJones, \& Kable, in press; Jeffries, 2007; Waxman, 2010). Including a skill review session prior to the simulation is another commonly practiced activity (Rochester et al., 2012), but there are other variations in and around the preparation for, and guidance during and following, the simulation encounter (Cant \& Cooper, 2010). Variation in itself should not be perceived as problematic but responsive to participants' specific learning needs. However, what remains unclear is the participant's or student's perceived benefits to their learning from the varied components of a simulation encounter.

In early work by Jeffries and colleagues (2007), a range of simulation evaluation instruments were created, specifically the Simulation Design Scale; Educational Practices in Simulation Scale and Student Satisfaction and Self-Confidence in Learning Instrument. Although these measures have been validated and used in subsequent studies (Kardong-Edgren, Adamson, \& Fitzgerald, 2010; Reese, Jeffries, \& Engum, 2010), other aspects of simulation delivery or the relationship of learning to clinical judgement are not overt within these and other instruments. Another recently developed measure, the Satisfaction with Simulation Experience Scale (Levett-Jones et al., 2011) 
connects aspects of the simulation (the facilitator, debriefing, reflection) to clinical reasoning but remains generic and does not include the full array of simulation components used across other settings. Further, there are few studies that focus on the relative value of components of a simulation encounter for learners from varied backgrounds. So the main aim of this study was to investigate the contribution of 11 specific simulation components to the enhancement of clinical judgement for students from three different study streams within an undergraduate nursing program.

\section{Theoretical Framework for Clinical Judgement}

At one large Australian university, simulation has been fully integrated into the renewed Bachelor of Nursing (BN) program. One theoretical framework embedded across the $\mathrm{BN}$ courses and related simulations is Tanner's Model of Clinical Judgment (2006). The model comprises four discrete aspects, which represent how expert nurses 'think' when engaging in patient care. The aspects of noticing, interpreting, responding and reflecting provide a schema for less experienced nurses to understand and develop their practice and for educators to use as a scaffold for teaching and learning. Key to students' understanding and prioritising patients' care requirements is honing their skills in noticing. What students bring to the patient care situation is based on prior exposure to similar scenarios and expectations of what might unfold, underpinned by theoretical knowledge and life experiences (Tanner, 2006). Simulations are one way to provide students exposure to patient care scenarios to contribute to noticing and clinical judgement.

\section{The Simulation}

In the final year of the Bachelor of Nursing, a deteriorating patient simulation has been included within a clinically focused medical-surgical course over the last five years. This simulation provides opportunity for students nearing the end of their degree to engage in an authentic post-operative surgical scenario and to interact with a 'patient', 'relative/s', and other team members. Students are given opportunity to assess (notice) the clinical situation (decreased urine output and oxygen 
saturations with increased breathlessness), determine appropriate actions within the context of the scenario and information available (interpret), and communicate with senior colleagues in response to the patient's deteriorating condition (respond).

The format of the simulation followed Jeffries' (2007) recommendations, and included a briefing and orientation to the simulation environment, the simulation activity and post simulation debriefing. Additional components were online access to patient case information one week prior to the simulation, specific questions for student observers to contribute to the debriefing session and the possibility for guidance by the academic during the simulation. A second academic resided in a separate 'control room' and provided patient responses via the manikin's speaker. This design and level of support were selected as the Faculty were in the early stages of implementing contemporary simulations and this was the first such experience for many of the students.

During one of the weekly clinical laboratory sessions, five to six students actively participated in a simulation role while five or six remaining students observed the $1^{\text {st }}$ phase of the patient scenario. On conclusion of the $1^{\text {st }}$ phase, student observers then swapped over and actively participated in the $2^{\text {nd }}$ phase while their peers became observers. The two phases represented two time points of the same patient case. The simulation ran for approximately 10 minutes and was followed by a 20 minute debriefing session facilitated by the academic. A post simulation survey was then used to gain students' insight about their learning experiences in relation to clinical judgement.

\section{Methods}

This is a quantitative descriptive study which examined nursing students' ratings of simulation components that contributed to clinical judgement. Subsequent to participation in the deteriorating patient simulation encounter described above, a convenience sample of final year students from six classes over two years $(n=150)$ were asked to participate in the research by completing a survey.

\section{Ethical considerations}


This study was granted approval by the university human research ethics committee. All students participated in the simulation as part of the respective course offering and those who agreed to engage in the research did so voluntarily. Students were aware there was no course credit from participating in the research. Each participant was provided with a survey code to de-identify responses. The primary author, who was not involved with teaching the respective classes, collected the data. Students could opt out of the research at any time without consequence.

\section{Post simulation survey - data management and analysis}

Demographic data collected included: age, gender, study stream, years of previous nursing experience, highest educational qualification, and number of previous simulations.

Student's rating of the benefit of individual simulation components to their clinical judgement was assessed using a survey developed for the study by the primary and secondary authors, both experts in simulation and education assessment. The survey questions addressed 11 components of the simulation determined through a literature review and based on the aspects of clinical judgement from Tanner's model. The survey was pilot tested on 30 students and modifications made to five questions. Participants were asked to rate each of 11 components of the simulation on the benefit the component had on applying clinical judgement using a 5 -point Likert Scale $(1=$ little assistance to 5 = great assistance). Not all 11 components assessed, such as guidance by the academic, are included in all simulation activities, but students would have experienced all 11 components at some stage in simulations during the two or three years of their study program. Students were familiar with Tanner's model through course lectures and previous clinical laboratory sessions.

Data were analysed using the SPSS (version19) computer software program. Data were summarised using frequencies and percentages for categorical data and means and standard deviations or medians and range for continuous data. Analysis of variance (ANOVA) was used to determine whether study stream (3-year, 2-year Enrolled Nurse, 2-year Graduate Entry), age, 
years of nursing experience, and gender influenced students' ratings of the benefit of the different components. The significance level was set at $\mathrm{p}<.05$.

\section{Findings}

One hundred and two from a possible 150 nursing students participated in the research (response rate $68 \%$ ). The sample comprised three student groups: recent school leavers undertaking a 3year nursing program (57\%); 2-year Graduate Entry (GE) students (25\%) who possessed a Bachelor degree in another discipline and were making a career change to nursing and 2-year Enrolled Nurse (EN) students (18\%) who had completed one year of technical college education, had prior clinical experience and were upgrading their qualifications.

The sample was predominantly female (82\%), aged $19-25$ years $(68.9 \%)$ and had two or less years of nursing experience (63\%). Over $70 \%$ of respondents had either one or no previous simulation encounters.

\section{Simulation Component Ratings and Ranking}

Participants' ratings on the assistance that the 11 simulation components provided to clinical judgement ranged from mean 3.23 to 4.02 (5-point rating scale) as illustrated in Table 1 . The three simulation components which received the highest ratings for contributing to clinical judgement and mean scores above 3.7 were: 1) facilitated debriefing, 2) post-simulation reflection, and 3) guidance by the academic. The components which participants rated least beneficial to clinical judgement were the patient case notes (mean 3.23), and participating in a role (mean 3.33).

\section{Comparison of Rankings by study program}

Table 2 illustrates the ratings students gave for the benefit of the simulation component to clinical judgement across the three student groups. The mean scores were all above 2.9 indicating that students thought all components assisted in some way. The top two ratings were the same for all groups: facilitated debriefing and post-simulation reflection, but the lowest ratings varied. The 
lowest ratings for 3-year students was patient case notes, for 2-year EN students the patient case scenario topic and for 2-year GE students participation in a role.

Statistically significant differences in mean ratings occurred in two simulation component areas: in post simulation reflection ( $\mathrm{F} 6.16 ; \mathrm{p}=.003)$ specifically the 3-year program mean score (3.74 SD 1.05) was lower than the 2-year GE (4.58 SD .78). The second statistically significant difference was viewing the simulation recording ( $F$ 5.245; $\mathrm{p}=.008)$, with the 3 -year program having a low mean (2.97 SD 1.19) compared with the 2-year GE students (4.3 SD .95). No other variable tested (age, years of nursing experience, or gender) had a statistically significant effect on the mean scores of simulation components.

\section{Discussion}

Developing and enhancing students' clinical decision making to form judgements about patients' care needs is a desired outcome for all nursing programs. Simulation is one teaching and learning method to enable a holistic, active experience for students to appreciate the range of registered nurse practices and responsibilities. This study provides new insight into three unique senior nursing student groups of an expanded range of simulation components and the level of assistance each provided in applying clinical judgement.

The high value of facilitated debriefing for these students corroborates existing literature of the value of debriefing for learning (K. T. Dreifuerst, 2009, 2011; Lusk \& Fater, 2013; Mariani, Cantrell, Meakim, Prieto, \& Dreifuerst, 2012) and similarly here, for applying clinical judgement. Many would agree that post simulation reflection commences and extends beyond the time allocated for debriefing (Kristina Thomas Dreifuerst, 2012; Shinnick, Woo, \& Mentes, 2011). Reflection was highly valued, ranked $2^{\text {nd }}$ to debriefing, with statistically significant differences across two of the student groups. Reflective practice is an important professional attribute to develop (Schön, 1995; Tanner, 2006) and it appears that simulation triggers reflection during and immediately following the simulation experience. 
The third highest rating component however is not always incorporated into the delivery of simulation activities - that of guidance by the academic. Practices vary in the level and manner of support provided to students during simulations and range from: no academic support (neither physical presence within the simulation room nor communication by phone), to proxy guidance through the manikin's responses or by phone (via the academic), to an academic physically taking on a role and actively engaging in the simulation scenario. For students who are advanced in their study program, guidance by the academic may be perceived as unnecessary. Yet this component was rated one of the top three for assisting students to make clinical judgements, likely because the group were novices in simulation. However, irrespective of the level of theoretical knowledge and skills practice, study findings support that students value interacting with or observing experienced teachers engaging in clinical practice learning activities, which simulation enables to a much greater degree (Aronson, Glynn, \& Squires, 2013; McGrath et al., 2012),. Although the study group were final year nursing students, most had limited simulation experiences so it was not surprising that despite having more knowledge, it appeared that senior students gained from the guidance provided during the simulation in applying clinical judgement.

Components rated least beneficial for clinical judgement were the patient case notes and briefing and orientation to the simulation area. Given the survey questions were focussed on clinical judgement, it is not surprising these components provided less value for students during the simulation. However these components offer important context for learning and should always be provided, particularly for students and those new to simulation. Although data about viewing the simulation AV playback were collected for one year only, there is marked contrast across student groups for this component. The perceived value of viewing the audiovisual playback (Chronister \& Brown, 2012) may vary depending on the student cohort as the 2-year GE group had much higher mean scores compared with the two other groups.

The findings from this ranking exercise are useful in that the student's perspective of what is considered important in their learning and application of clinical judgement becomes clearer, rather 
than what academics assume may be of most value within simulation activities. The key insights from these data are that student's value facilitated debriefing, reflection and guidance by the experienced academic for assisting with clinical judgement. Although differences emerged of the relative value of components across the three groups, above average ratings for all components indicated benefit for these students. Further, it appears that regardless of age, years of nursing experience or gender, simulation is similarly beneficial for clinical judgement across different student cohorts therefore tailoring is not necessary.

\section{Study strengths and limitations}

These particular findings have not been reported previously in the literature and provide a valuable perspective from both pedagogical and operational perspectives to consider when developing and delivering simulations. The response rate of $68 \%$ provides reasonable representation of the majority opinion but a higher rate would be desirable. The survey requires use in different populations to determine psychometric properties. Self-report as a single level of inquiry has limitations in reliability related to social desirability. Although the survey was conducted on the same day as the simulation, groups crossed over from participation to observer roles and would have had opportunity to begin to reflect on their own and others' practice. Study findings therefore report immediate impressions and early perceptions of the post-simulation reflective process. Data obtained from multi- rather than single-site institutions would provide more generalisable findings.

\section{Conclusion}

Opinion from students with varying backgrounds about the components that 'matter most' sheds new light for simulation practice in relation to application of clinical judgement. Irrespective of the entry level or program of study, this student population rated all 11 simulation components useful in applying clinical judgement. Tanner's model provides a scholarly framework for curricula, simulations and enhancing students' clinical judgement for nursing practice. 


\section{References}

Aronson, B., Glynn, B., \& Squires, T. (2013). Effectiveness of a Role-Modeling Intervention on Student Nurse Simulation Competency. Clinical Simulation in Nursing, 9(4), e121-e126. doi: 10.1016/j.ecns.2011.11.005

Arthur, C., Levett-Jones, T., \& Kable, A. (in press). Quality indicators for the design and implementation of simulation experiences: A Delphi study. Nurse Education Today. doi: 10.1016/j.nedt.2012.07.012

Cant, R., \& Cooper, S. (2010). Simulation-based learning in nurse education: Systematic review. Journal of Advanced Nursing, 66(1), 3-15. doi: 10.1111/j.1365-2648.2009.05240.x

Chronister, C., \& Brown, D. (2012). Comparison of Simulation Debriefing Methods. Clinical Simulation in Nursing, 8(7), e281-e288. doi: 10.1016/j.ecns.2010.12.005

Dreifuerst, K. T. (2009). The essentials of debriefing in simulation and learning: A concept analysis. Nursing Education Perspectives, 30(2), 109-114.

Dreifuerst, K. T. (2011). Debriefing for Meaningful Learning(:) A Reflective Strategy to Foster Clinical Reasoning. Clinical Simulation in Nursing, 7(6), e250. doi:

10.1016/j.ecns.2011.09.023

Dreifuerst, K. T. (2012). Using Debriefing for Meaningful Learning to Foster Development of Clinical Reasoning in Simulation. Journal of Nursing Education, 51(6), 326-333. doi: 10.3928/01484834-20120409-02

Jeffries, P. (Ed.). (2007). Simulation in nursing education: from conceptualization to evaluation. New York: National League for Nursing.

Kardong-Edgren, S., Adamson, K. A., \& Fitzgerald, C. (2010). A Review of Currently Published Evaluation Instruments for Human Patient Simulation. Clinical Simulation in Nursing, 6(1), e25-e35. doi: 10.1016/j.ecns.2009.08.004

Laschinger, S., Medves, J., Pulling, C., McGraw, R., Waytuck, B., Harrison, M. B., \& Gambeta, K. (2008). Effectiveness of simulation on health profession students' knowledge, skills, confidence and satisfaction. International Journal of Evidence-Based Healthcare, 6(3), 278(225).

Levett-Jones, T., McCoy, M., Lapkin, S., Noble, D., Hoffman, K., Dempsey, J., . . Roche, J. (2011). The development and psychometric testing of the Satisfaction with Simulation Experience Scale. Nurse Education Today, 31, 705-710. doi: 10.1016/j.nedt.2011.01.004 
Lusk, J. M., \& Fater, K. (2013). Postsimulation Debriefing to Maximize Clinical Judgment Development. Nurse Educator January/February, 38(1), 16-19.

Mariani, B., Cantrell, M. A., Meakim, C., Prieto, P., \& Dreifuerst, K. T. (2012). Structured Debriefing and Students' Clinical Judgment Abilities in Simulation. Clinical Simulation in Nursing. doi: 10.1016/j.ecns.2011.11.009

McGaghie, W., Issenberg, S. B., Petrusa, E. R., \& Scalese, R. J. (2010). A critical review of simulation-based medical education research: 2003-2009. Medical Education, 44(1), 5063. doi: 10.1111/j.1365-2923.2009.03547.x

McGrath, M., Lyng, C., \& Hourican, S. (2012). From the Simulation Lab to the Ward: Preparing 4th Year Nursing Students for the Role of Staff Nurse. Clinical Simulation in Nursing, 8(7), e265-e272. doi: 10.1016/j.ecns.2010.10.003

Miller, K., K. , Riley, W., Davis, S., \& Hansen, H., E. . (2008). In Situ Simulation: A Method of Experiential Learning to Promote Safety and Team Behavior. Journal of Perinatal \& Neonatal Nursing, 22(2), 105.

Neill, M. A., \& Wotton, K. (2011). High-Fidelity Simulation Debriefing in Nursing Education: A Literature Review. Clinical Simulation in Nursing, 7(5), e161-e168. doi:

10.1016/j.ecns.2011.02.001

Reese, C. E., Jeffries, P., \& Engum, S. A. (2010). Learning together: using simulations to develop nursing and medical student collaboration. Nursing Education Perspectives, 31(1), 33-37.

Ricketts, B. (2011). The role of simulation for learning within pre-registration nursing education - A literature review. Nurse Education Today, 31(7), 650-654. doi: 10.1016/j.nedt.2010.10.029

Rochester, S., Kelly, M., Disler, R., White, H., Forber, J., \& Matiuk, S. (2012). Providing simulation experiences for large cohorts of 1 st year nursing students: Evaluating quality and impact. Collegian, 19(3), 117-124. doi: 10.1016/j.colegn.2012.05.004

Schlairet, M. (2011). Simulation in an Undergraduate Nursing Curriculum: Implementation and Impact Evaluation. Journal of Nursing Education, 50(10), 561.

Schön, D. (1995). The reflective practitioner: how professionals think in action. Aldershot: Avebury Ashgate Publishing Ltd.

Shinnick, M. A., Woo, M. A., \& Mentes, J. C. (2011). Human Patient Simulation: State of the Science in Prelicensure Nursing Education. Journal of Nursing Education, 50(2), 65-72. doi: 10.3928/01484834-20101230-01 
Tanner, C. A. (2006). Thinking like a nurse: A research-based model of clinical judgment in nursing. Journal of Nursing Education, 45(6), 204 - 221.

Waxman, K. T. (2010). The Development of Evidence-Based Clinical Simulation Scenarios: Guidelines for Nurse Educators. Journal of Nursing Education, 49(1), 29. doi: 10.3928/01484834-20090916-07 